PROCEEDINGS OF THE

AMERICAN MATHEMATICAL SOCIETY

Volume 126, Number 3, March 1998, Pages 907-911

S $0002-9939(98) 04104-5$

\title{
A COUNTEREXAMPLE TO A $B P$-ANALOGUE OF THE CHROMATIC SPLITTING CONJECTURE
}

\author{
ETHAN S. DEVINATZ
}

(Communicated by Thomas Goodwillie)

\begin{abstract}
We prove that, if $n \geq 2$, the $E(n-1)_{*}$-localization of the $K(n)_{*-}$ localization map $B P_{p} \rightarrow L_{K(n)} B P$ is not a split monomorphism in the stable category by exhibiting spectra $Z$ for which the map $\pi_{*}\left(L_{n-1}\left(B P_{p}\right) \wedge Z\right) \rightarrow$ $\pi_{*}\left(L_{n-1}\left(L_{K(n)} B P\right) \wedge Z\right)$ is not injective. If $p \geq \max \left\{\frac{1}{2}\left(n^{2}-2 n+2\right), n+1\right\}$ and $n \geq 3$, we show that $Z$ may be taken to be a two-cell complex in the sense of $E(n-1)_{*}$-local homotopy theory. The question of whether the map $L_{n-1}\left(B P_{p}\right) \rightarrow L_{n-1} L_{K(n)} B P$ splits was asked by Hovey and is in some sense a $B P$-analogue of Hopkins' chromatic splitting conjecture.
\end{abstract}

\section{INTRODUCTION}

Let $E$ be a spectrum, and let $\iota_{E}(X): X \rightarrow L_{E} X$ denote the $E_{*}$-localization of the spectrum $X$. If $E=E(n)$, write $\iota_{n}$ and $L_{n}$ for $\iota_{E(n)}$ and $L_{E(n)}$, where $E(n)$ is the Landweber exact theory with coefficient ring $E(n)_{*}=\mathbb{Z}_{(p)}\left[v_{1}, \ldots, v_{n}, v_{n}^{-1}\right]$. As usual, $p$ is a fixed prime number. Now let $K(n)$ denote the $n^{\text {th }}$ Morava $K$-theory; its coefficient ring is $\mathbb{F}_{p}\left[v_{n}, v_{n}^{-1}\right]$. Hopkins' chromatic splitting conjecture (see $[2,4]$ for a complete discussion) asserts that the map

$$
L_{n-1} \iota^{\wedge}\left(S_{p}^{0}\right): L_{n-1}\left(S_{p}^{0}\right) \rightarrow L_{n-1} L_{K(n)} S^{0}
$$

is a split monomorphism in the stable category, where we write $X_{p}$ for the $p$ completion of the spectrum $X$ and $\iota^{\wedge}$ for $\iota_{K(n)}$. (Since the map $S^{0} \rightarrow S_{p}^{0}$ is a $K(n)_{*}$-equivalence for all $n \geq 1, L_{K(n)} S^{0}=L_{K(n)} S_{p}^{0}$.) More specifically, it asserts that $L_{n-1} L_{K(n)} S^{0}$ splits as a wedge of certain suspensions of copies of $L_{k}\left(S_{p}^{0}\right)$ with $k \leq n-1$. The conjecture is known to be true for $n=1$ and for $n=2$ with $p>3$. For $n>2$, essentially nothing is known. An important consequence of this conjecture would be the result that if $f: X \rightarrow Y$ is a map between ( $p$-localizations of) finite spectra such that $L_{K(n)} f: L_{K(n)} X \rightarrow L_{K(n)} Y$ is trivial for each $n$, then $f$ is itself trivial. This is relevant to the generating hypothesis (see [1]), as are some other consequences.

In [2], Hovey proves that if $f$ is as above, then the composition $X \stackrel{f}{\rightarrow} Y \rightarrow B P \wedge Y$ is trivial. He asks whether the map

$$
L_{n-1} \iota^{\wedge}\left(B P_{p}\right): L_{n-1}\left(B P_{p}\right) \rightarrow L_{n-1} L_{K(n)} B P
$$

Received by the editors May 7, 1996 and, in revised form, August 30, 1996.

1991 Mathematics Subject Classification. Primary 55N22, 55Q10.

Partially supported by the National Science Foundation. 
is the inclusion of a wedge summand, since this would also imply his result. We will call this question the $B P$-analogue of the chromatic splitting conjecture, although the reader should be warned that Hovey uses this terminology for the result above that he proved.

Of course, the chromatic splitting conjecture does not imply that $L_{n-1} \iota^{\wedge}\left(B P_{p}\right)$ is a split monomorphism, since $K(n)$ is not smashing in the sense of $[5,1.28]$. In fact, the general statement of the chromatic splitting conjecture is inconsistent with the statement that the canonical map $B P \wedge L_{K(n)} S^{0} \rightarrow L_{K(n)} B P$ is an equivalence.

In this note, we prove that the $B P$-analogue of the chromatic splitting conjecture is false for $n \geq 2$ by finding spectra $Z$ such that

$$
\pi_{*}\left(L_{n-1} \iota^{\wedge}\left(B P_{p}\right) \wedge Z\right): \pi_{*}\left(L_{n-1}\left(B P_{p}\right) \wedge Z\right) \rightarrow \pi_{*}\left(L_{n-1} L_{K(n)}(B P) \wedge Z\right)
$$

is not a monomorphism. (By Corollary 1.2, $\pi_{*} L_{n-1} \iota^{\wedge}\left(B P_{p}\right)$ is a monomorphism. Thus the $B P$-analogue of the chromatic splitting conjecture holds for $n=1$, since any map between rationally local spectra which induces a monomorphism on $\pi_{*}$ is the inclusion of a wedge summand.) We will also find "minimal counterexamples" for $n \geq 3$ and $p \geq \max \left\{\frac{1}{2}\left(n^{2}-2 n+2\right), n+1\right\}$, and we will show that, for $n=2$, one cannot find such small counterexamples. Although we believe that parts of the chromatic splitting conjecture are true - for example we believe that $L_{n-1} L_{K(n)} X$ is as predicted when $X$ is an $E(n-1)_{*}$-acyclic finite spectrum - we wonder whether these minimal counterexamples might somehow be related to the failure of the general chromatic splitting conjecture for $n \geq 3$.

\section{A counterexample}

The following easy lemma will be the main tool for constructing our counterexamples.

Lemma 1.1. Let $Z$ be any spectrum, and suppose $n \geq 1$. Then

$$
\pi_{*}\left(L_{n-1} \iota^{\wedge}\left(B P_{p}\right) \wedge Z\right)
$$

is a monomorphism if and only if $B P_{p *} L_{n-1} Z$ is $v_{n}$-torsion free.

Proof. The map $\pi_{*}\left(L_{n-1} \iota^{\wedge}\left(B P_{p}\right) \wedge Z\right)$ can be rewritten as

$$
\iota^{\wedge}\left(B P_{p}\right)_{*}\left(L_{n-1} Z\right): B P_{p *}\left(L_{n-1} Z\right) \rightarrow\left(L_{K(n)} B P\right)_{*}\left(L_{n-1} Z\right)
$$

since $E(n-1)$ is smashing $[6,7.5 .6]$.

Recall that [2]

$$
\left(L_{K(n)} B P\right)_{*}=\varliminf_{I=\left(i_{0}, \ldots, i_{n-1}\right)} v_{n}^{-1} B P_{*} /\left(p^{i_{0}}, \ldots, v_{n-1}^{i_{n-1}}\right)
$$

and is therefore flat over $v_{n}^{-1} B P_{*}$. Hence

$$
\left(L_{K(n)} B P\right)_{*} \otimes_{B P_{*}} B P_{*} X \rightarrow\left(L_{K(n)} B P\right)_{*} X
$$

is an isomorphism for any spectrum $X$. Furthermore, the Landweber exact functor theorem [3] (applied to $\left(L_{K(n)} B P\right)_{*} / v_{n}^{-1}\left(B P_{p}\right)$ ) implies that $v_{n}^{-1} B P_{p^{*}} X \rightarrow$ $\left(L_{K(n)} B P\right)_{*} X$ is a monomorphism for any spectrum $X$. Since $\iota^{\wedge}\left(B P_{p}\right)_{*}\left(L_{n-1} Z\right)$ factors as

$$
B P_{p^{*}} L_{n-1} Z \rightarrow v_{n}^{-1} B P_{p *} L_{n-1} Z \rightarrow\left(L_{K(n)} B P\right)_{*} L_{n-1} Z,
$$

we have that $\iota^{\wedge}\left(B P_{p}\right)_{*} L_{n-1} Z$ is a monomorphism if and only if $B P_{p *} L_{n-1} Z \rightarrow$ $v_{n}^{-1} B P_{p *} L_{n-1} Z$ is a monomorphism. This proves the lemma. 
Corollary 1.2. $\pi_{*}\left(L_{n-1} \iota^{\wedge}\left(B P_{p}\right)\right)$ is a monomorphism.

Proof. Since $E(n-1)$ is smashing, $B P_{*} L_{n-1} S^{0}=\pi_{*} L_{n-1} B P$ as $B P_{*}$-modules. Moreover,

$$
\pi_{*} L_{n-1} B P \approx \begin{cases}B P_{*} \otimes \mathbb{Q}, & n=1, \\ B P_{*} \oplus \Sigma^{-n+1} B P_{*} /\left(p^{\infty}, v_{1}^{\infty}, \ldots, v_{n-1}^{\infty}\right), & n>1,\end{cases}
$$

as $B P_{*}$-modules $[5,6.2]$. Thus $B P_{p *} L_{n-1} S^{0}$ has no $v_{n}$-torsion; the use of Lemma 1.1 now completes the proof.

We now exhibit a spectrum $Z$ such that $B P_{p *} L_{n-1} Z$ has nontrivial $v_{n}$-torsion and thus disprove the $B P$-analogue of the chromatic splitting conjecture.

Proposition 1.3. Let $v_{n}: \Sigma^{2\left(p^{n}-1\right)} B P \rightarrow B P$ be the BP-module map inducing multiplication by $v_{n}$ on $\pi_{*} B P$, and let $Z$ be its cofiber. If $n \geq 2, B P_{p *} L_{n-1} Z$ has nontrivial $v_{n}$-torsion.

Proof. It suffices to find an element $x$ in $B P_{p *} L_{n-1} B P$ such that

$$
v_{n} x \in \operatorname{im} B P_{p *}\left(L_{n-1} v_{n}\right) \text { but } x \notin \operatorname{im} B P_{p *}\left(L_{n-1} v_{n}\right) .
$$

Begin by observing that $B P_{p *} L_{n-1} B P$ is an algebra over $B P_{p *} B P$ via the map $B P_{p *}\left(\iota_{n-1}\right)$ and that $B P_{p *} L_{n-1} v_{n}$ is just multiplication by $\eta_{R}\left(v_{n}\right) \in B P_{p *} B P$. Since $E(n-1)$ is smashing, we have the following commutative diagram:

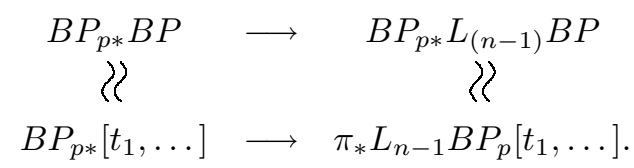

The bottom map is just the map on polynomial algebras induced by the localization map $B P_{p *} \rightarrow \pi_{*} L_{n-1} B P_{p}$.

Now let

$$
x=1 / p v_{1} \cdots v_{n-1} \in \pi_{*} L_{n-1} B P_{p} \subset \pi_{*} L_{n-1} B P_{p}\left[t_{1}, \ldots\right]=B P_{p *} L_{n-1} B P,
$$

where we have identified $\pi_{*} L_{n-1} B P_{p}$ as in (1.1). Since

$$
\eta_{R}\left(v_{n}\right)=v_{n} \quad \bmod \left(p, v_{1}, \ldots, v_{n-1}\right),
$$

it follows that $v_{n} x=\eta_{R}\left(v_{n}\right) x$; hence $v_{n} x \in \operatorname{im}\left(B P_{p *} L_{n-1} v_{n}\right)$. But we also have $\eta_{R}\left(v_{n}\right)=v_{n} \bmod \left(t_{1}, \ldots\right)$; it is then easily seen from diagram (1.2) that $x \notin$ $\operatorname{im}\left(B P_{p *} L_{n-1} v_{n}\right)$. This completes the proof.

\section{A minimal Counterexample}

We have seen that $\pi_{*}\left(L_{n-1} \iota^{\wedge}\left(B P_{p}\right)\right)$ is a monomorphism. The next result shows that if $n \geq 3$ and $p \geq \max \left\{\frac{1}{2}\left(n^{2}-2 n+2\right), n+1\right\}$, then there exist 2-cell complexes $Z$ (in the sense of $E(n-1)_{*}$-local homotopy theory $\left.[1,1]\right)$ such that

$$
\pi_{*}\left(L_{n-1} B P_{p} \wedge Z\right) \rightarrow \pi_{*}\left(L_{n-1} L_{K(n)}(B P) \wedge Z\right)
$$

is not a monomorphism. Thus, in these cases, the $B P$-analogue of the generating hypothesis fails almost immediately.

Proposition 2.1. Let $n \geq 3$ and $p \geq \max \left\{\frac{1}{2}\left(n^{2}-2 n+2\right), n+1\right\}$. Then there exists a map $g: L_{n-1} S^{0} \rightarrow L_{n-1} S^{0}$ whose cofiber has nontrivial $v_{n}$-torsion. 
Remark 2.2. $g$ is actually a map from a suspension of $L_{n-1} S^{0}$ to $L_{n-1} S^{0}$. For the rest of the paper we will suppress suspensions from the notation if no confusion is likely to result.

Proof. Recall from $[4,5]$ the elements $x_{n-1, n-1} \in v_{n-1}^{-1} B P_{*}$ defined by

$$
x_{n-1, n-1}= \begin{cases}\left(x_{n-1,1}\right)^{p^{n-2}}-v_{n-2}^{p^{n-1}-1} v_{n-1}^{p^{n-1}-p^{n-2}+1}, & n \geq 4, \\ \left(x_{2,1}\right)^{p}-v_{1}^{p^{2}-1} v_{2}^{(p-1) p+1}-v_{1}^{p^{2}+p-1} v_{2}^{p^{2}-2 p} v_{3}, & n=3,\end{cases}
$$

where

$$
x_{n-1,1}=v_{n-1}^{p}-v_{n-2}^{p} v_{n-1}^{-1} v_{n} .
$$

By $[4,5.10], x_{n-1, n-1}$ is invariant $\bmod \left(p, v_{1}, \ldots, v_{n-3}, v_{n-2}^{p^{n-1}+p-1}\right)$. In particular,

$$
x_{n-1, n-1} / p v_{1} \cdots v_{n-3} v_{n-2}^{p^{n-1}+1} \in H^{0}\left(v_{n-1}^{-1} B P_{*} /\left(p^{\infty}, \ldots, v_{n-2}^{\infty}\right)\right),
$$

where, if $M$ is a $B P_{*} B P$-comodule, we write $H^{*} M$ for $\operatorname{Ext}_{B P_{*} B P}^{*}\left(B P_{*}, M\right)$. Now

$$
B P_{*} M_{n-1} S^{0}=v_{n-1}^{-1} B P_{*} /\left(p^{\infty}, \ldots, v_{n-2}^{\infty}\right),
$$

where $M_{n-1} S^{0}$ is defined as in [5, 5]. By sparseness and the fact that

$$
H^{i}\left(B P_{*} M_{n-1} S^{0}\right)=0
$$

for $i \geq(n-1)^{2}$ (see [1, A1.5]), it follows from the convergence results of [6, Chapter $8]$ that the element in (2.1) survives to an element $g_{0}$ in $\pi_{*} M_{n-1} S^{0}$. Define $g$ so that the diagram

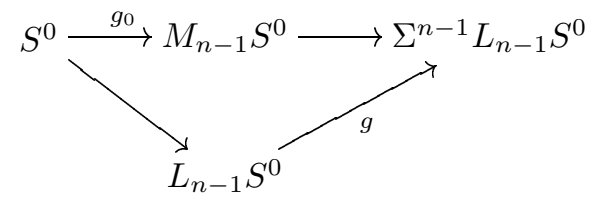

commutes, where the map $M_{n-1} S^{0} \rightarrow \Sigma^{n-1} L_{n-1} S^{0}$ is the fiber of the canonical $\operatorname{map} \Sigma^{n-1} L_{n-1} S^{0} \rightarrow \Sigma^{n-1} L_{n-2} S^{0}$.

We now prove that the cofiber of $g$ has nontrivial $v_{n}$-torsion by finding an element $z$ in $B P_{p *} L_{n-1} S^{0}$ with $v_{n}^{p^{n}-2} z \in \operatorname{im}\left(B P_{p *} g\right)$ but $z \notin \operatorname{im}\left(B P_{p *} g\right)$. Indeed, $g$ is a map of degree $-(n-1) \bmod 2(p-1)$, so it follows from sparseness and the description of $B P_{*} L_{n-1} S^{0}$ in (1.1) that

$$
\begin{aligned}
\operatorname{im}\left(B P_{p *} g\right)=\operatorname{im}\left(B P_{p *} S^{0} \stackrel{B P_{p *} g_{0}}{\longrightarrow} B P_{p *} M_{n-1} S^{0}\right. & \rightarrow B P_{p *} \Sigma^{n-1} L_{n-1} S^{0} \\
& \left.\rightarrow B P_{p *} /\left(p^{\infty}, \ldots, v_{n-1}^{\infty}\right)\right) .
\end{aligned}
$$

But the composition

$$
B P_{p *} M_{n-1} S^{0} \rightarrow B P_{p *} \Sigma^{n-1} L_{n-1} S^{0} \rightarrow B P_{p *} /\left(p^{\infty}, \ldots, v_{n-1}^{\infty}\right)
$$

is just the usual map $v_{n-1}^{-1} B P_{p *} /\left(p^{\infty}, \ldots, v_{n-2}^{\infty}\right) \rightarrow B P_{p *} /\left(p^{\infty}, \ldots, v_{n-1}^{\infty}\right)$; therefore, $\operatorname{im}\left(B P_{p *} g\right)$ is the $B P_{p *}$-submodule of $B P_{p *} /\left(p^{\infty}, \ldots, v_{n-1}^{\infty}\right)$ generated by $v_{n}^{p^{n-2}} / p v_{1} \cdots v_{n-2} v_{n-1}^{p^{n-2}}$. Let $z \in B P_{p *} L_{n-1} S^{0}$ correspond to $1 / p v_{1} \cdots v_{n-2} v_{n-1}^{p^{n-2}}$. One can then show that $v_{n}^{p^{n-2}} z \in \operatorname{im}\left(B P_{p *} g\right)$ but $z \notin \operatorname{im}\left(B P_{p *} g\right)$, completing the proof.

We conclude this paper by observing that Proposition 2.1 is not true if $n=2$. 
Proposition 2.3. Let $p$ be an odd prime, and let $g: \Sigma^{k} L_{1} S^{0} \rightarrow L_{1} S^{0}$ be any map. Then the cofiber of $g$ is $v_{2}$-torsion free.

Proof. We may regard $g \in \pi_{k}\left(L_{1} S^{0}\right)$. Recall that $[5,8.10]$

$$
\pi_{k} L_{1} S^{0}= \begin{cases}\mathbb{Z}_{(p)}, & k=0, \\ \mathbb{Q} / \mathbb{Z}_{(p)}, & k=-2, \\ \mathbb{Z} /\left(p^{m+1}\right), & k=2(p-1) s p^{m}-1, \quad p \nmid s, \\ 0, & \text { otherwise. }\end{cases}
$$

In degree $2(p-1) s p^{m}-1, \pi_{k} L_{1} S^{0}$ is generated by the map $\alpha_{s p^{m} / m+1}$ given by the composition

$$
L_{1} S^{k+1} \rightarrow \Sigma^{k+1} L_{1} M\left(p^{m+1}\right) \stackrel{v_{1}^{s p^{m}}}{\longrightarrow} L_{1} M\left(p^{m+1}\right) \rightarrow L_{1} S^{1}
$$

where $M\left(p^{m+1}\right)$ denotes the $\bmod \left(p^{m+1}\right)$ Moore spectrum, and $v_{1}^{s p^{m}}$ is a selfmap inducing multiplication by $v_{1}^{s p^{m}}$ on $B P_{*} L_{1} M\left(p^{m+1}\right)$. The left end map is the inclusion of the bottom cell, and the right end map is the projection onto the top cell. In degree $-2, \alpha_{p^{m} / m+1} \alpha_{-p^{m} / m+1}$ generates the subgroup of elements of order $p^{m}$.

If $k=0$, the cofiber of $g$ is just the $E(1)_{*}$-localization of a Moore spectrum and hence is $v_{2}$-torsion free. Next suppose $g=c \alpha_{s p^{m} / m+1}, c \in \mathbb{Z} /\left(p^{m+1}\right)$. By the same argument as in the proof of Proposition 2.1, we have

$$
\begin{aligned}
& \operatorname{im}\left(B P_{p *} g\right)=\operatorname{im}\left(B P_{p *} \rightarrow v_{1}^{-1} B P_{p *} /\left(p^{m+1}\right) \stackrel{c v_{1}^{s p^{m}}}{\longrightarrow} v_{1}^{-1} B P_{p *} /\left(p^{m+1}\right)\right. \\
&\left.\longrightarrow P_{p *} /\left(p^{\infty}, v_{1}^{\infty}\right)\right) .
\end{aligned}
$$

If $s>0, \operatorname{im}\left(B P_{p *} g\right)=0$, so the cofiber of $g$ is evidently $v_{2}$-torsion free. If $s<0$, it is easy to see that $v_{2} z \in \operatorname{im}\left(B P_{p *} g\right)$ if and only if $z \in \operatorname{im}\left(B P_{p *} g\right)$, so that the cofiber of $g$ is again $v_{2}$-torsion free. Finally, if $g \in \pi_{-2} L_{1} S^{0}$, then $g$ is the composite of two maps, one of which induces the zero homomorphism on $B P_{p *} L_{1} S^{0}$. Hence the cofiber of $g$ is $v_{2}$-torsion free in this case as well.

\section{REFERENCES}

[1] E. S. Devinatz, The generating hypothesis revisited, to appear in Stable and Unstable Homotopy, Fields Institute Communications, Amer. Math. Soc., 1997.

[2] M. Hovey, Bousfield localization functors and Hopkins' chromatic splitting conjecture, The Cech Centennial, Contemp. Math., vol. 181, Amer. Math. Soc., Providence, Rhode Island, 1995, pp. 225-250. MR 96m:55010

[3] P. S. Landweber, Homological properties of comodules over $M U^{*} M U$ and $B P^{*} B P, A m e r$. J. Math. 98 (1976), 591-610. MR 54:11311

[4] H. R. Miller, D. C. Ravenel, and W. S. Wilson, Periodic phenomena in the Adams-Novikov spectral sequence, Ann. of Math. 106 (1977), 469-516. MR 56:16626

[5] D. C. Ravenel, Localization with respect to certain periodic homology theories, Amer. J. Math. 106 (1984), 351-414. MR 85k:55009

[6] D. C. Ravenel, Nilpotence and Periodicity in Stable Homotopy Theory, Ann. of Math. Stud. 128, Princeton University Press, Princeton, 1992. MR 94b:55015

Department of Mathematics, University of Washington, Box 354350, Seattle, WashINGTON 98195-4350

E-mail address: devinatz@math.washington.edu 